\begin{tabular}{|c|c|c|}
\hline $\begin{array}{c}\text { PRAMANA } \\
\text { journal of } \\
\text { physics }\end{array}$ & (c) Indian Academy of Sciences & $\begin{array}{l}\text { Vol. } 71, \text { No. } 4 \\
\text { October } 2008 \\
\text { pp. } 623-628\end{array}$ \\
\hline
\end{tabular}

\title{
Fission, spallation or fusion-based neutron sources
}

\author{
KURT N CLAUSEN \\ Paul Scherrer Institut, WBBA/122, CH-5232 Villigen PSI, Switzerland \\ E-mail: kurt.clausen@psi.ch
}

\begin{abstract}
In this paper the most promising technology for high power neutron sources is briefly discussed. The conclusion is that the route to high power neutron sources in the foreseeable future is spallation - short or long pulse or even CW - all of these sources will have areas in which they excel.
\end{abstract}

Keywords. Neutron sources; spallation; fission; inertial confined fusion.

PACS Nos 29.25.Dz; 25.40.Sc; 61.80.Hg; 28.20.Gd; 52.57.-2

To monitor the development of large scale facilities, attempts have been made to define figures of merit, which can be used to characterize progress in the source development. For X-ray sources the highest achievable brightness at a given source is often quoted as such an indicator (see figure 1). The brightness is a relatively good measure of an X-ray source as it is a measure of the beam quality, which can be seen at an actual instrument. The traditional neutron source is a research reactor and for irradiation purpose the figure of merit was the neutron flux at a given accessible position in the reactor. For beam reactors the traditional number to quote is the thermal flux at a position where a moderator can be placed or which can be viewed from a beam channel. The thermal flux is a well-defined number for a reactor. For non-fission sources, spallation- or fusion-based, the initial fast spectrum is different from a fission spectrum. A moderator is not needed for the neutron production itself, but only necessary to convert the fast spectrum into a useful spectrum for neutron scattering and the mode of operation is often pulsed rather than CW. The thermal flux or peak thermal flux is therefore not a welldefined, easily measurable quantity, but is based on an estimate or a simulation and therefore often named as an 'effective thermal flux'. Figure 2 shows one such attempt to compare the estimated effective thermal flux at fission- and spallationbased facilities. The pulsed sources have been marked by a line spanning the range between the time average flux and the estimated peak flux, to indicate that in actual experiments the useful flux is - depending on the experiment - somewhere between the time average and the peak flux. Recent advances in neutron optics and instrumentation have actually shown that in many cases it is advantageous to use long neutron pulses, i.e. sacrifice peak flux for more integrated and time-averaged flux (for the same construction and operating costs the obtainable time average 
Kurt $N$ Clausen

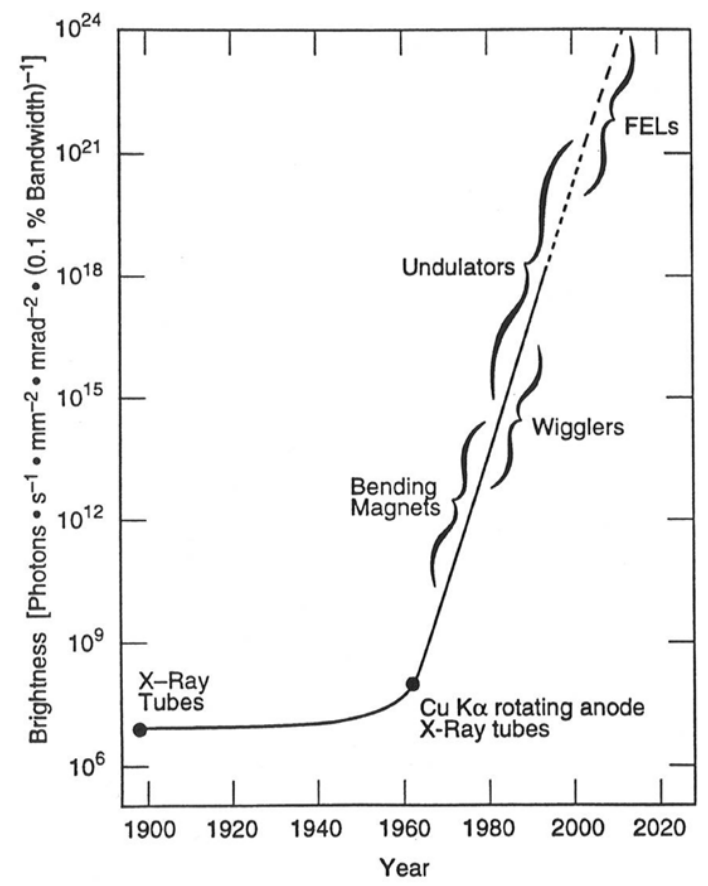

Figure 1. The development of the brightness of X-ray facilities (compilation from Friso van der Veen Paul Scherrer Institut, Villigen, CH).

neutron flux of a spallation source will increase markedly at each step, when going from short pulse to long pulse as in the extreme case to CW operation).

Of the tremendous progress in science based on neutrons since the first demonstration experiments by Shull and Brockhouse [1] in the 50th till today, only approximately an order of magnitude stems from the source, i.e., from the increased thermal flux. In the high flux reactors ILL, HFBR and HFIR operational since the early seventies and the pulsed spallation source ISIS from the mid-eighties, the predominant progress is due to the development of moderators, guides, beam optics, instrumentation, software and detectors. The next step in source performance is being realized just now in the new pulsed MW spallation sources coming on line, SNS in the US and J-SNS at J-PARC in Japan.

In a recent article [2], ideas for a substantial 2-3 orders of magnitude gain in effective thermal flux, using a fusion-based neutron source was discussed. The article was inspired by recent progress in laser technology and indicated a time frame for a possible realization between 2030 and 2040. In the following paragraphs the limits for sources based on fusion, spallation and proposed fusion-based facility are briefly discussed.

In table 1 a number of neutron producing nuclear reactions are listed. All the processes above fission can be utilized for small neutron sources, but are not feasible for high flux facilities because of the huge amount of associated release of heat. For fission-based neutron sources the performance is mainly limited by the associated heat generation, i.e., the maximum power density which can be kept cooled. 
Fission, spallation or fusion-based neutron sources

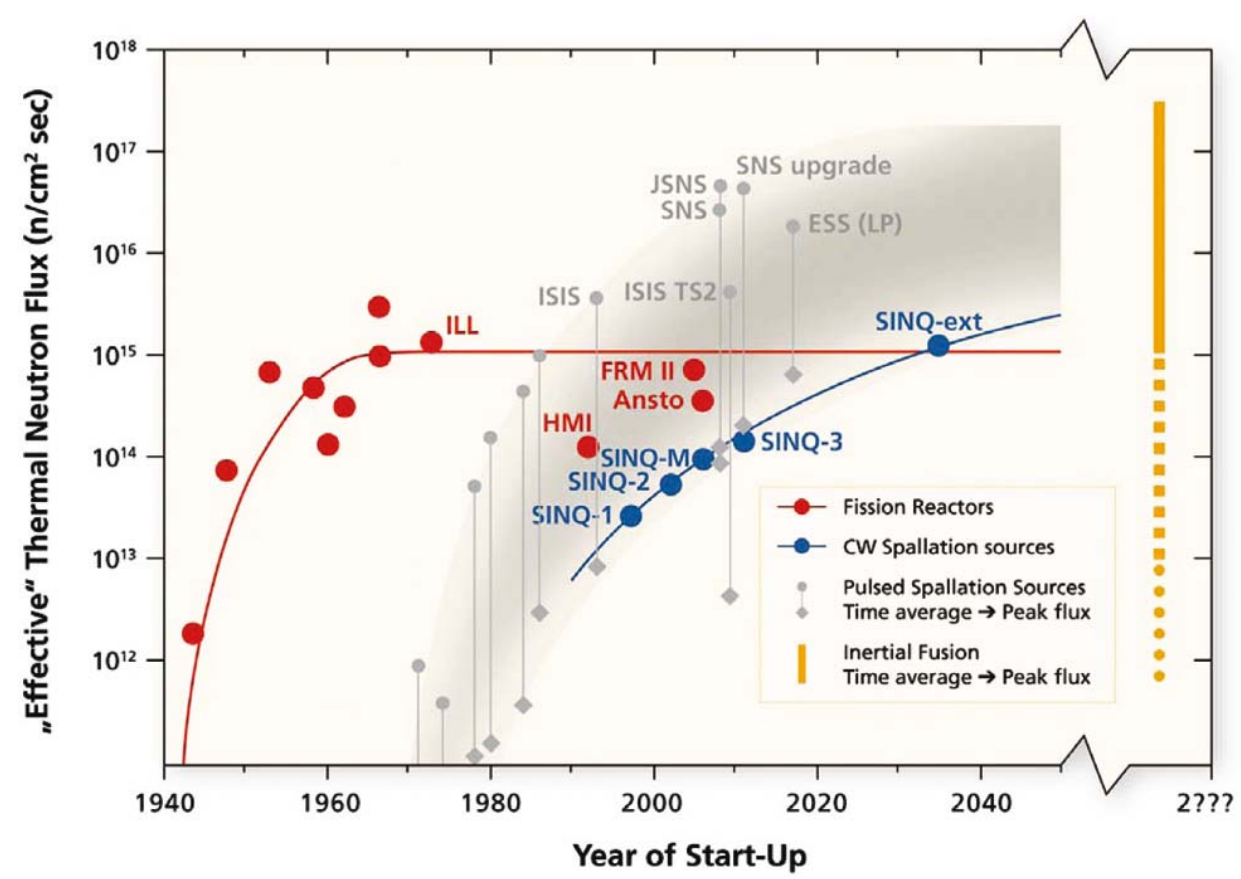

Figure 2. Thermal flux at the position where moderators can be placed for different types of neutron sources. The whole 'instrument' from moderator to neutron detector determines how effective these neutrons can be utilized. For the pulsed sources this - depending on the experiment - spans the grey band from time average to peak flux. The continuous spallation sources can be more easily compared to reactors, and extrapolating from the experience gained through the development of the SINQ facility we would be able to reach the thermal flux of ILL, with a 10 MW cyclotron-based facility (SINQ-ext in the figure). Pulsed spallation sources will probably be able to approach similar time-averaged fluxes.

For spallation sources the main limiting factor is a combination of coolable power density of the target and radiation damage in the target window, where the high energy proton beam enters the target. The latter depends on the time structure of the proton pulse. For very high power, liquid metal targets are necessary for cooling purposes. For these liquid metal targets measures must be taken to mitigate the effect of shock pulses in pulsed spallation sources. The expected limitation for fission- and spallation-based facilities are shown in figure 2 .

Inertial fusion does not have a problem with the target window, or heat release in the target as a tiny target capsule with deuterium-tritium explodes during each pulse. The energy generated in the process is however carried away with the generated high energy neutrons and $\alpha$-particles (see below). The $\alpha$-particles will both erode the wall/window around the source and provide a huge heat load on this surface. Inertial fusion devices are therefore meant to have a liquid metal first wall. 
Kurt $N$ Clausen

Table 1. Neutron yield and heat release for different nuclear reactions (compilation of data from $\mathrm{G}$ Bauer [3]).

\begin{tabular}{|c|c|c|c|}
\hline Nuclear process & Example & Neutron yield & $\begin{array}{l}\text { Heat release } \\
(\mathrm{MeV} / \mathrm{n})\end{array}$ \\
\hline $\mathrm{D}-\mathrm{T}$ in solid target & $\begin{array}{l}400 \mathrm{keV} \text { deuterons }(\mathrm{D}) \\
\text { on tritium }(\mathrm{T}) \text { in } \mathrm{Ti}\end{array}$ & $4 \times 10^{-5} \mathrm{n} / \mathrm{D}$ & 10.000 \\
\hline Deuteron stripping & $\begin{array}{l}40 \mathrm{MeV} \text { deuterons } \\
\text { on liquid } \mathrm{Li}\end{array}$ & $7 \times 10^{-2} \mathrm{n} / \mathrm{D}$ & 3.500 \\
\hline $\begin{array}{l}\text { Nuclear photoeffect } \\
\text { from } \mathrm{e}^{-} \text {-brems- } \\
\text { strahlung }\end{array}$ & $100 \mathrm{MeV}$ e ${ }^{-}$on ${ }^{238} \mathrm{U}$ & $5 \times 10^{-2} \mathrm{n} / \mathrm{e}^{-}$ & 2.000 \\
\hline${ }^{9} \mathrm{Be}(\mathrm{p}, \mathrm{n} ; \mathrm{p}, \mathrm{pn})$ & $11 \mathrm{MeV}$ protons $(\mathrm{p})$ on $\mathrm{Be}$ & $5 \times 10^{-3} \mathrm{n} / \mathrm{p}$ & 2.000 \\
\hline${ }^{9} \mathrm{Be}(\mathrm{D}, \mathrm{n}){ }^{10} \mathrm{Be}$ & $15 \mathrm{MeV} \mathrm{D}$ on $\mathrm{Be}$ & $1 \mathrm{n} / \mathrm{D}$ & 1.000 \\
\hline Nuclear fission & $\begin{array}{l}\text { Fission of }{ }^{235} \mathrm{U} \\
\text { by thermal neutrons }\end{array}$ & $1 \mathrm{n} /$ fission & 180 \\
\hline Spallation & $800 \mathrm{MeV}$ p on ${ }^{238} \mathrm{U}$ on $\mathrm{Pb}$ & $\begin{array}{l}27 \mathrm{n} / \mathrm{p} \\
17 \mathrm{n} / \mathrm{p}\end{array}$ & $\begin{array}{l}55 \\
30\end{array}$ \\
\hline Inertial fusion & $\begin{array}{l}\mathrm{D}-\mathrm{T} \text { pellets compressed } \\
\text { by powerful lasers }\end{array}$ & $1 \mathrm{n} / \mathrm{D}-\mathrm{T}$ pair & $\begin{array}{l}\text { Target }=\text { pellet } \\
\text { explodes each pulse }\end{array}$ \\
\hline
\end{tabular}

In the proposed source the heat carried away by $\alpha$-particles and neutrons amount to $300 \mathrm{MW}$, and it will be about an order of magnitude higher if one follows the red dotted line in figure 1 of the article by Taylor et al [2]. To solve this problem, the authors propose to have a first wall in front of each moderator in the form of a 'conveyor belt' of lead bricks -1 brick $(2.5 \mathrm{~kg}$ lead) per pulse per moderator which at $10 \mathrm{~Hz}$ amounts to 2160 metric tons of (radioactive) lead per moderator that need to be handled daily. This is one of the many technical difficulties with the inertial fusion neutron sources.

The second problem for the inertial fusion device is the high initial energy of the produced neutrons $(14.1 \mathrm{MeV})$ which is substantially higher than the majority of neutrons in a fission or spallation spectrum (both distributions peak around 1-2 $\mathrm{MeV}$ ). The scattering cross-sections for $\mathrm{H}$ and other relevant moderating materials drop considerably when the neutron energy is increased from 1 to $14 \mathrm{MeV}$. In figure 3 the scattering cross-section for $\mathrm{H}$ is shown. Bearing in mind that the number density for hydrogen in water is $0.067 \AA^{-3}$ and the cross-section for 14.1 $\mathrm{MeV}$ neutrons is $c a 0.8 \mathrm{~b}$ (figure 3 ) this corresponds to a moderation length of the order of $1 /\left(0.8 \times 10^{-24} \mathrm{~cm}^{2} \times 0.067 \times 10^{24} \mathrm{~cm}^{-3}\right) \sim 19 \mathrm{~cm}$, which is at least a factor 3-4 larger than an acceptable moderator size. As it is difficult to foresee better moderating materials in this environment, it can be concluded that an inertial fusion device will be inefficiently moderated. 
Fission, spallation or fusion-based neutron sources

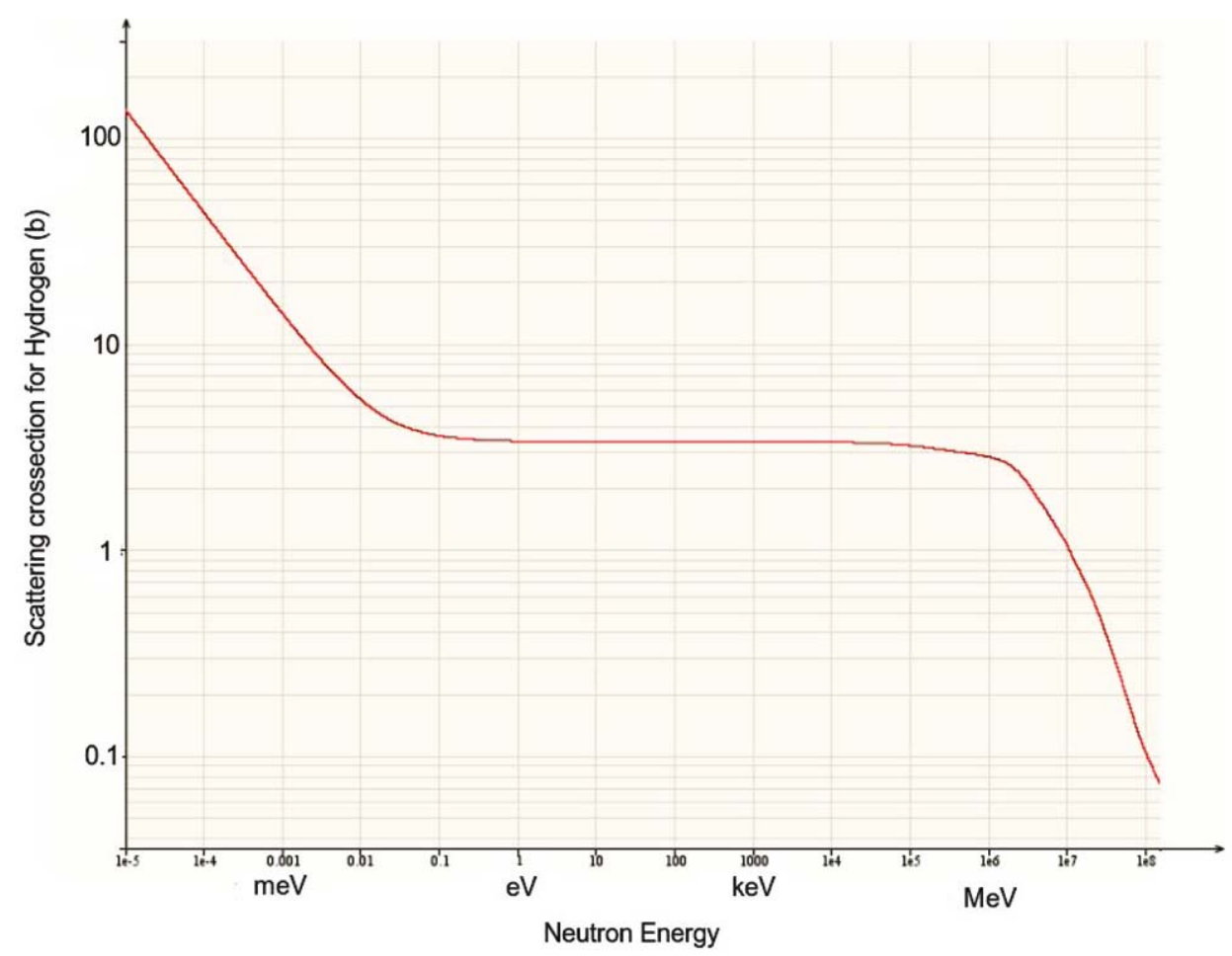

Figure 3. Neutron cross-section for H. Just above $1 \mathrm{MeV}$ there is a sharp decrease in the scattering cross-section (data from G Bauer) [3].

The third problem is the fabrication of the $\mathrm{D}-\mathrm{T}$ pellets and the handling of large amounts of tritium. Each day 864.000 encapsulated complex D-T pellets must be either fabricated on site or transported to the site. The annual turnover of tritium is $10 \mathrm{~kg}$, which by any standards is a huge quantity and a very serious proliferation issue.

If the first wall is placed at a distance compatible with tolerable irradiation damage to wall and moderator construction materials, then the flux at the moderator position will at most be similar to the conditions at an optimized spallation source, i.e. an inertial fusion-based facility cannot be expected to outperform a spallationbased facility.

The final argument that will be touched upon in this paper is the indicated time schedule. A prototype inertial fusion facility, which can handle $10 \mathrm{~Hz}$ repetition rates is not even considered yet - the worldwide fusion activities are focused on the realization of ITER (thermonuclear fusion). Experience have shown (see figure 2) that the time from first demonstration to a dedicated source designed for neutron scattering is ca 30 year. Demonstration of a $10 \mathrm{~Hz}$ inertial fusion facility is more likely to be 30 years away, i.e. a source based on this technology even further away. Most important is however that the technical risks are enormous and that a fusion-based facility is not expected to outperform or be more cost-effective than a spallation source. This in combination with a number of serious issues, such 
as proliferation (handling $>10 \mathrm{~kg}$ of tritium per year), licensing as a user facility, unsolved technology and waste problems with the initial fusion technology, means that the route for high power neutron sources in the foreseeable future is spallation - short or long pulse or even CW - all of these sources will have areas in which they excel.

\section{References}

[1] Thomas Mason, Masatoshi Arai and Kurt N Clausen, MRS Bulletin 28(12), 923 (2003)

[2] Andrew Taylor et al, Science 315, 1092 (2007)

[3] Data compiled for the ESS project 2000-2003 by G Bauer, Forschungszentrum Jülich, Germany 